Mots. Les langages du politique

\title{
Proximité éditoriale : normes et usages des genres journalistiques
}

\section{Roselyne Ringoot et Yvon Rochard}

\section{(2) OpenEdition \\ Journals}

Édition électronique

URL : https://journals.openedition.org/mots/162

DOI : $10.4000 /$ mots. 162

ISSN : 1960-6001

Éditeur

ENS Éditions

Édition imprimée

Date de publication : 1 mars 2005

Pagination : 73-90

ISBN : 2-84788-077-1

ISSN : 0243-6450

Référence électronique

Roselyne Ringoot et Yvon Rochard, «Proximité éditoriale : normes et usages des genres journalistiques », Mots. Les langages du politique [En ligne], 77 | 2005, mis en ligne le 31 janvier 2008 consulté le 23 avril 2022. URL : http://journals.openedition.org/mots/162 ; DOI : https://doi.org/ $10.4000 /$ mots. 162

\section{(C) ENS Éditions}




\section{Proximité éditoriale : normes et usages des genres journalistiques}

La proximité, en journalisme, est érigée en loi. Parfois formalisée dans des guides du journalisme, mais plus souvent transmise et véhiculée de façon informelle au sein des rédactions, la loi de proximité conditionne des choix éditoriaux et des pratiques de terrain; «la loi du mort-kilomètre ( $^{1}$ en est un exemple galvaudé. Dans le travail présenté ici, nous considèrerons la proximité à la fois en tant que facteur de production du discours et en tant que construction du discours. Il s'agit donc de définir les critères et les composantes de la proximité journalistique et, par ailleurs, de dégager les effets de sens de la proximité. Nous démontrerons que plusieurs formes de proximité caractérisent le journalisme, ce qui amène à ne pas restreindre le terme de "proximité» à sa seule acception spatiale. Par «proximité éditoriale», nous entendons l'ensemble des mécanismes discursifs créant de la proximité entre le journal et son lecteur. Cette proximité éditoriale s'articule sur deux niveaux de pertinence : la proximité infrastructurelle liée aux processus d'écriture journalistique et plus particulièrement celui des genres journalistiques, et la proximité circonstancielle variable en fonction des centres d'intérêt développés par les lignes éditoriales.

Nous proposons donc dans un premier temps une théorie énonciative des genres journalistiques inspirée des travaux de Dominique Maingueneau développant la notion d'ethos. Partant du principe que «chaque genre de discours comporte une distribution préétablie des rôles qui détermine en partie l'image de soi du locuteur $»^{2}$, nous dégagerons comment les genres journalistiques préconstruisent la relation de proximité entre le journal et son lecteur et comment la mise en scène du journaliste dans les textes induit des formes différentes de la proximité.

1. Les accidents mortels sont d'autant mieux traités qu'ils concernent le lectorat du journal : lieu de l'accident et/ou identité des victimes.

2. P. Charaudeau, D. Maingueneau, 2002, Dictionnaire d'analyse de discours, Paris, Le Seuil. 
Nous aborderons ensuite la question de la proximité liée aux variables de la ligne éditoriale en étudiant le cas de magazines régionaux relevant d'une double contrainte éditoriale : la proximité géographique et la proximité thématique. Le corpus comprend trois magazines bretons analysés du point de vue des régularités thématiques (nature de l'information traitée) et des régularités discursives (manière dont l'information est traitée). Si la ligne éditoriale de ces magazines postule un lien communautaire régional, le rapport au territoire ne se construit pas comme dans la presse dite «de proximité» qui quadrille son périmètre de diffusion en microterritoires locaux (villes, communes, cantons, départements, en presse quotidienne ou hebdomadaire régionale). Ces périodiques revendiquent une identité bretonne, ce qui induit une proximité qui n'est pas strictement territoriale. Le politique, l'économique et le culturel, qui sont les champs informatifs de prédilection d'Armor, Nouvel Ouest et ArMen, sont ancrés dans une perception identitaire de la Bretagne et nous verrons comment les success-stories relatées par les trois titres produisent des effets d'appartenance.

Cette démonstration articulée sur les propriétés intrinsèques des genres journalistiques et sur leurs usages en fonction du projet éditorial fondé ici sur le sentiment régional met en évidence les interactions à l'œuvre dans la proximité éditoriale.

\section{Proximité et production de l'information}

Dans son Manuel de journalisme, Yves Agnès 3 décline la loi de proximité selon quatre axes : proximité géographique, chronologique, psychoaffective et sociétale. Ces quatre entrées s'articulent sur un noyau central qui est la figure du lecteur dont on évalue le potentiel d'intérêt pour l'information en fonction de ce qui lui est proche. Ainsi, l'axe géographique propose une échelle de valeurs entre la rue et l'Europe passant par le quartier, la ville, le pays socioculturel, la région, le département, le pays. L'axe chronologique va de l'aujourd'hui/demain à l'hier, après-demain/avant-hier. L'axe psychoaffectif échelonne la sécurité, l'argent, la famille, les enfants, les loisirs, la santé, la sexualité, alors que, sur l'axe "vie en société», se succèdent travail/éducation/maison, loisirs/associations, religion/politique. Nous ne discuterons pas ici cette échelle de valeurs susceptible d'évaluer le proche et lointain du lecteur, mais nous soulignons le caractère essentiel de la proximité appréhendée dans une approche technique. Si cette vision de la proximité n'est pas toujours aussi rationalisée, elle n'en est pas moins effective dans les pratiques routinières de

3. Y. Agnès, 2002, Manuel de journalisme, Écrire pour le journal, Paris, La Découverte. 
la production d'information. Selon les lignes éditoriales, des croisements sont opérés de manière à construire une communauté de lecture plus ou moins élargie en fonction du cumul des variables : communauté professionnelle ou d'intérêt dans la presse spécialisée, communauté sexuée dans la presse dite féminine et/ou masculine ${ }^{4}$, communauté générationnelle (presse jeunesse, presse seniors), communauté géoculturelle en presse régionale... Nous considérons donc la proximité comme un préconstruit fondateur du discours journalistique. Concrètement, le potentiel de proximité supposée va déterminer la sélection des sujets informatifs et leur traitement : angles, format, genres, positionnement dans le journal, choix organisationnels (qui va traiter l'information, avec quels moyens et dans quel ordre de priorité). La notion de proche et de lointain est un véritable instrument de mesure conditionnant des décisions (refus ou prescription d'un sujet par la hiérarchie), une distribution des tâches, une dimension économique (le profil du lecteur est utilisé par les annonceurs en termes de ciblage).

La proximité érigée en loi a pour corollaire le culte du terrain que le journaliste partage avec le sociologue et l'homme politique : «Le fétichisme du terrain en sociologie aujourd'hui présente de troublantes analogies avec le mythe de la proximité en politique. Lorsqu'un acteur politique parle du terrain, se décrit comme homme de terrain, appelle ses homologues à venir voir sur le terrain, évoque l'action qu'il mène concrètement, loin des bureaux des ministères, au plus près du terrain, il active des dichotomies fondamentales qui ont cours à peu près à l'identique en sociologie. Dans les deux cas, le propos s'articule autour d'une croyance fondamentale, selon laquelle la réalité est accessible à partir du terrain. ${ }^{5}$

La notion de terrain en journalisme est tout aussi fondamentale qu'en politique ou en sociologie depuis que la presse d'information a pris le pas sur la presse d'opinion à la fin du $19^{\mathrm{e}}$ siècle. Denis Ruellan définit le reportage, «figure de proue du journalisme qui l'incarnait tout entier depuis 1880 », comme une "pratique d'inspiration anglo-saxonne qui rencontra en France les méthodes du courant littéraire à la mode, le naturalisme [...] et dont l'originalité - pour l'époque - était de partir de l'observation de terrain, de témoignages, des faits et de leurs discours pour apporter au lecteur un éclairage sur la réalité en train de se faire, sur le monde en mouvement ${ }^{6}$. Ce rapport au terrain va déterminer la pratique professionnelle du journaliste en articulant la perception

4. Sur l'articulation communauté sexuée et discours journalistique, voir B. Damian, G. Soulez, 2001, "L'alcôve et la couette ", Réseaux, vol. 19, n 105.

5. C. Le Bart, 2002, "La proximité politique comme légitimité d'en bas», introduction à l'atelier «proximité politique», C. Le Bart et R. Lefebvre (dir.), Congrès de l’AFSP, Lille.

6. D. Ruellan, 1997, Les "pro» du journalisme, de l'état au statut. La construction d'un espace professionnel, Presses universitaires de Rennes, p. 93. 
du réel en situation de recherche d'informations et le processus d'écriture en situation de rédaction :

Le modèle américano-anglais de journalisme institutionnalise peu à peu un ensemble de règles d'écriture intériorisées par les journalistes, contrôlées par les rédacteurs en chef. Il s'agit de l'objectivité comme revendication d'un récit vérifié et neutre des faits, séparation du fait et du commentaire. La règle des cinq W (Who ? What? When ? Where? Why ?) détermine les contenus obligés de tout reportage?

On voit là poindre l'aporie de l'objectivité journalistique fondée sur une technique d'observation et d'écriture du réel, donc sur autre chose que le réel en soi. D'une part, la technicité discursive littéraire et politique qui marque les écrits journalistiques jusqu'à la fin du $19^{\mathrm{e}}$ siècle est minimisée, d'autre part, la technicité discursive journalistique est affirmée par la fameuse règle. Cependant, cette règle institue le terrain tout autant qu'elle permet d'en rendre compte. Nous situons donc l'émergence du fait journalistique sur le pivot discursif $^{8}$ qui fait interagir une perception préconstruite du réel et une représentation structurée 9 . Revendiquée et pratiquée par les journalistes, la règle des cinq W n'est pourtant pas proclamée comme instituant le terrain. Elle est plutôt convoquée dans une dimension analogique, c'est-à-dire en tant qu'instrument de reproduction du réel (et non de production) : «les faits sont les faits ». Terrain et empirie sont confondus alors que la mise en discours marque la rupture entre les deux; paradoxalement, la nécessaire affirmation de la technicité journalistique va de pair avec la négation de son caractère constructif ou plutôt de sa dimension constructiviste ${ }^{10}$. Cette croyance à un réel réductible au discours qui en rend compte est ancrée dans l'idéologie de la proximité journalistique qui abolit la distance entre les mots et les choses. La fonction de miroir social attribuée aux journaux en est une déclinaison, dans la mesure où la propriété spéculaire conférée au journalisme est une version radicalisée de la proximité. Cela dit, journalisme de terrain et journalisme de plume ne s'excluent pas l'un l'autre : soit ils cohabitent au sein d'un même journal, soit ils investissent des supports différents, articulant ainsi proximité physique et proximité d'esprit.

7. É. Neveu, 2001, Sociologie du journalisme, Paris, La Découverte, p. 63.

8. Voir les présupposés épistémologiques de la posture défendue ici dans R. Ringoot, «Discours journalistique : analyser le discours de presse au prisme de la ligne éditoriale», dans R. Ringoot, P. Robert-Demontrond, 2004 (dir.), L'analyse de discours, Rennes, séminaires méthodologiques de l'IREIMAR, Éditions Apogée.

9. Le réel est à la fois la matière première et le produit transformé : R. Ringoot, 2002, «Périodicité et historicité de l'info en ligne», Médiamorphoses, $n^{\circ}$ 4, p. 69-74.

10. R. Barthes, 1985, L'aventure sémiologique, Paris, Le Seuil, p. 95 : «La rhétorique est une technè; (ce n'est pas une empirie), c'est-à-dire : le moyen de produire une des choses qui peuvent indifféremment être ou n'être pas, dont l'origine est dans l'agent créateur, non dans l'objet créé; il n'y a pas de technè de choses naturelles et nécessaires : le discours ne fait donc partie ni des unes ni des autres.» 


\section{Genres journalistiques et proximité}

La notion de genres journalistiques, pour autant qu'elle soit utilisée par les professionnels de l'information et par les chercheurs, n'est pas une notion stable. La catégorisation la plus courante dans les guides du journalisme distingue les genres d'information (brève, filet, compte rendu, reportage, interview...) et les genres de commentaire (éditorial, billet, chronique...).

Mais il est difficile de comprendre la (les) logique(s) de classements proposés par les manuels de journalisme. Les définitions varient et les catégories se chevauchent. Cette complexité et les différences s'expliquent par les croisements de critères qui vont des choix stylistiques microlinguistiques aux intentions communicatives, en passant par la position énonciative du locuteur et le contenu des articles.

Ce constat est formulé par Jean-Michel Adam ${ }^{11}$ à partir de l'étude de quatre manuels dans lesquels il recense vingt-quatre genres identifiés. Tout en conservant le clivage information-commentaire, le linguiste propose une approche énonciative selon deux positions polaires : le pôle distance-information et le pôle implication-commentaire. La distance opposée à l'implication concerne à la fois la position du journaliste et le type de traitement de l'information. Dans une autre étude discursive menée sur les genres, Guy Lochard développe une approche fonctionnaliste en croisant la visée communicationnelle des genres (informative, persuasive, séductrice, factitive) et les modes dominants (argumentatif, descriptif, énonciatif, narratif). Cet éclairage sociocommunicatif qui renvoie à la relation journaliste/lecteur articule aussi deux positions en tension rappelant le couple information-commentaire, que l'auteur formule par l'opposition entre le pôle objectivité/légitimité et le pôle subjectivité/crédibilité ${ }^{12}$.

L'autre catégorisation des genres en "genres assis» et «genres debout» est issue de la sociologie du journalisme. La distinction d'un journalisme assis (confiné à la rédaction) et d'un journalisme debout (de contact) ${ }^{13}$ a donné, par extension, l'opposition entre genres liés au terrain et genres liés au travail de bureau. L'éditorial, le commentaire, le billet, la chronique, présupposent un travail sédentaire, un référent documentaire. Le reportage, mais aussi l'interview, le portrait, l'enquête, le compte rendu présupposent un terrain et des contacts physiques, un référent situationnel de l'information. Bien sûr, concrètement, les frontières ne sont pas étanches. Dans les pratiques afférentes à l'écriture tout comme dans l'écriture elle-même, le travail de terrain présuppose une

11. J.-M. Adam, 1997, "Unités rédactionnelles et genres discursifs : cadre général pour une approche de la presse écrite ", Pratiques, n ${ }^{94}$, p. 3-18.

12. G. Lochard, 1996, "Genres rédactionnels et appréhension de l'évènement médiatique. Vers un déclin des "modes configurants" ?", Réseaux, $n^{\circ}$ 76, p. 83-102.

13. Voir le rappel de ces notions dans É. Neveu, Sociologie du journalisme, ouvr. cité. 
préparation et le travail de bureau ne se limite pas à l'écriture; référents documentaires et référents situationnels peuvent être convoqués et mixés dans tous les écrits. Cependant, les genres impliquent des attendus où, dans un cas, la proximité est articulée sur la médiation physique opérée par le rédacteur, «l'avoir été là » de Roland Barthes ${ }^{14}$; dans l'autre cas, la proximité est articulée sur la médiation idéelle. Nous retrouvons le terrain de l'action opposé au terrain des idées.

En problématisant les genres journalistiques par la proximité, nous proposons une autre approche radicalisant une position énonciativiste mais ancrée dans une perspective socioprofessionnelle. Considérés comme processus d'engendrement du discours ${ }^{15}$, les genres journalistiques peuvent s'appréhender en fonction de l'ethos. Pour Maingueneau,

l'énonciateur doit légitimer son dire : dans son discours, il s'octroie une position institutionnelle et marque son rapport à un savoir. Mais il ne se manifeste pas seulement comme un rôle et un statut, il se laisse aussi appréhender comme une voix et un corps. Aussi l'ethos se traduit-il dans le ton, qui se rapporte aussi bien à l'écrit qu'au parlé, et qui s'appuie sur une double figure de l'énonciateur, celle d'un caractère et d'une corporalité ${ }^{16}$.

Nous proposons donc trois classes de genres à partir des stratégies énonciatives mettant en scène la figure du journaliste : les genres corporalisants, les genres caractérisants, les genres dépersonnalisants. Tous trois conditionnent l'écriture et la lecture du journal (professionnalisme du journaliste et horizon d'attente du lecteur) en fonction de trois formes de proximité différentes élaborées selon trois techniques discursives.

\section{Genres corporalisants}

Dans ce cadre, nous regroupons les genres qui induisent une mise en scène du journalisme en train de se faire, en signifiant la corporalité du journaliste ${ }^{17}$ et/ou de ses sources. L'interview est le cas majeur de cette double incarnation avec le jeu des questions/réponses mimant l'entretien vécu. L'objectif du portrait est de mettre en scène une personne choisie, mais la présence induite du journaliste est tout aussi importante : marques évaluatives dans les descrip-

14. R. Barthes, 1982, L'obvie et l'obtus. Essais critiques III, Paris, Le Seuil.

15. Cette terminologie fait référence au programme coopératif «Hybridation et création de genres médiatiques » initié dans le cadre du Réseau d'étude sur le journalisme. Les travaux portant sur neuf terrains, menés par vingt-sept chercheurs nationaux et internationaux, donneront lieu à deux publications aux Presses universitaires de Rennes : R. Ringoot, J.-M. Utard (dir.), à paraitre en 2005 et D. Augey, F. Demers (dir.), à paraitre en 2006.

16. P. Charaudeau, D. Maingueneau, 2002, ouvr. cité, p. 239.

17. Nous nous inspirons ici de l'analyse du discours militaire de C. Oger, 2000, "De l'esprit de corps au corps du texte : cohésion militaire et dissolution journalistique », Langage et société, n 94, décembre, p. 9-43. 
tions, ambiance, imbrication de citations renvoyant à l'entretien... Le reportage doit transporter le lecteur dans l'action par la médiation du journaliste, aussi sa propre mise en scène est-elle incontournable. Le compte rendu et l'enquête proposent une figure du journaliste plus nuancée. Dans l'enquête, la monstration du journaliste à l'œuvre est double : d'une part, l'expression du journaliste sur le terrain avec des descriptions et des citations d'informateurs; d'autre part, la figure du journaliste sur le terrain documentaire (éléments statistiques, documents cités ou reproduits...). Le compte rendu renvoie à de multiples variables, mais nous le définirons dans la tension entre représentation du terrain prouvant la présence du journaliste et effacement énonciatif.

Dans ces genres rédactionnels, la corporalité énonciative du journaliste est l'enjeu de la proximité induite pour le lecteur. L'écriture construit un «avoir été là » du journaliste qui agit comme pivot d'identification. Le corps écoutant, parlant, bougeant du journaliste dans le texte est un corps transubstantiel! Les genres liés au terrain et à l'objectivité qui l'auréole convoquent le corps en mouvement du journaliste dans l'écrit.

\section{Genres caractérisants}

L'éditorial, le commentaire, l'analyse, la chronique ou le billet ne passent pas par la mise en scène physique du journalisme en train de se faire qui correspond au travail sur les ressources documentaires, aux concertations avec ses pairs ou ses sources... Cependant, comme dans toute énonciation, ces genres construisent « une image de soi du locuteur». L'implication du journaliste signifiée dans le texte est une implication qui renvoie davantage à l'intellectualité. Le mouvement mis en scène dans ces textes est un mouvement du dit et du pensé (argumentation), et du ressenti (humeur, prise de position), du caractère selon la terminologie de Maingueneau. La proximité induite pour le lecteur est une proximité d'esprit et de sentiment. Pour reprendre les termes de Barthes, ce n'est plus «l'avoir été là» qui compte, mais «l'être là» dans le discours. Il s'agit de construire une communauté de compréhension et d'interprétation entre journaliste et lecteurs faisant appel aux schémas discursifs de la persuasion, de l'explication ou de l'adhésion. Les humeurs du billet, tout comme les positionnements de l'éditorial, les avis prodigués dans la chronique, les éclairages apportés par l'analyse sont aussi soumis à des traitements énonciatifs construisant une image mentale du journaliste et du lecteur. L'activité intellectuelle et l'engagement induits par le genre ne signifient pas qu'il soit mis en scène de façon homogène : l'éditorial qui est lié à l'expression de l'opinion peut relever d'une stratégie discursive privilégiant la neutralité ${ }^{18}$ ou l'implication.

18. R. Koren, 2003, «Enjeu et pratique de l'engagement neutre : le cas de l'écriture de presse», dans Argumentation et prise de position : pratiques discursives, Sémen, $\mathrm{n}^{\circ} 17$. 
Ces genres dits nobles ne tirent pas leur prestige du terrain, mais de la hauteur qu'ils induisent : position élevée (englobante et réflexive) par rapport à l'information et l'actualité, et position élevée dans la hiérarchie professionnelle ${ }^{19}$. Réservés à des rédacteurs illustres qui symbolisent le journal, les genres caractérisants induisent une proximité par l'individualisation de la relation journaliste-lecteur. La notoriété de l'auteur et la régularité des écrits provoquent un effet de retrouvailles au fil des numéros : le ton, le style, le positionnement intellectuel, idéologique ou émotionnel construisent une attente personnalisée.

\section{Genres dépersonnalisants}

Dans le cas des genres corporalisants tout comme dans le cas des genres caractérisants, la scénarisation de la personne ou la personnalité du journaliste favorise la complicité du sens et des sens. À ceci s'ajoute le facteur de la signature qui concrétise l'individuation. Hormis l'éditorial qui est (ou a été) parfois ramené à l'énonciateur collectif (le nom du journal), ces genres sont signés. Que faire alors des genres marqués par l'impersonnel de l'énonciation, tels que la brève, le filet ou la mouture ? Dans le clivage traditionnel entre information et commentaire, ils sont regroupés dans la famille des genres dits informatifs avec l'interview, le reportage, le portrait, l'enquête, le compte rendu. Dans l'approche assis-debout, le desk (brève, filet, mouture) ${ }^{20}$ est classé dans le journalisme assis, avec l'éditorial, la chronique, le billet... Dans notre approche, ces genres ne relèvent ni du processus énonciatif de corporalisation, ni du processus de caractérisation, ils correspondent même à leur contraire. Nous proposons donc une troisième catégorie regroupant les genres simulant la disparition de l'énonciateur. Avec l'effacement des traces énonciatives, l'information apparait comme non médiatée. Cette «disparition du journaliste » peut aussi être associée à l'effacement énonciatif des sources. La neutralisation de la figure du journaliste crée une absence mettant le lecteur en prise directe avec l'information traitée d'un point de vue utilitaire. Car c'est bien l'utilité de l'information dépouillée de tout magistère journalistique qui est ainsi privilégiée :

19. Cette opposition axiologique du haut (sommet d'un art et d'une carrière) et du bas (l'ancrage du terrain) ne casse pas pour autant le mythe du terrain évoqué en amont. Dans l'imaginaire journalistique, cette représentation verticale est associée à celle d'un parcours scalaire : c'est le passage par le bas qui légitime l'arrivée en haut.

20. La brève est un article court, de quelques dizaines de mots, qui répond de manière succincte mais précise aux questions de référence : qui, quoi, où, quand et, dans la mesure du possible, comment et pourquoi. Très proche de la brève, le filet répond aussi aux questions de référence, mais il développe l'information et l'explicitation (notamment le comment et le pourquoi). Sa longueur peut être cinq fois supérieure à celle de la brève. La mouture est une synthèse plus documentée que le filet. Elle est réalisée à partir de multiples sources : dépêche, documents divers, déclaration d'expert, conférence de presse. D’après Y. Agnès, 2002, ouvr. cité. 
débarrassée des apparats du réalisme de terrain ou des méandres du commentaire, l'information est plus proche du lecteur.

La proximité peut alors se décliner selon trois stratégies énonciatives : incorporation du lecteur dans l'avoir été là du journaliste créant une proximité physique et émotionnelle, intégration du lecteur dans l'être là journalistique créant une proximité intellectuelle et émotionnelle, face à face entre lecteur et information créant une proximité fonctionnelle dénuée d'émotion. Soit le tableau suivant :

\begin{tabular}{|l|l|l|l|l|}
\hline Genre d'ethos & $\begin{array}{l}\text { Mise en scène } \\
\text { du journaliste (J) }\end{array}$ & $\begin{array}{l}\text { Convocation du } \\
\text { lecteur }\end{array}$ & $\begin{array}{c}\text { Genres } \\
\text { rédactionnels }\end{array}$ & $\begin{array}{c}\text { Genre } \\
\text { de proximité }\end{array}$ \\
\hline $\begin{array}{l}\text { corporalisa- } \\
\text { tion }\end{array}$ & $\begin{array}{l}\text { du corps, } \\
\text { du faire }\end{array}$ & $\begin{array}{l}\text { dans l'action } \\
\text { avec le J }\end{array}$ & $\begin{array}{l}\text { reportage, } \\
\text { interview, } \\
\text { portrait, } \\
\text { enquête, CR }\end{array}$ & $\begin{array}{l}\text { physique } \\
\text { (+ émotion- } \\
\text { nelle) }\end{array}$ \\
\hline caractérisation & $\begin{array}{l}\text { du pensé, } \\
\text { du ressenti }\end{array}$ & $\begin{array}{l}\text { dans un dia- } \\
\text { logue virtuel } \\
\text { avec le J }\end{array}$ & $\begin{array}{l}\text { commentaire, } \\
\text { édito, billet, } \\
\text { chronique }\end{array}$ & $\begin{array}{l}\text { intellectuelle } \\
\text { (+ émotion- } \\
\text { nelle) }\end{array}$ \\
\hline $\begin{array}{l}\text { dépersonnali- } \\
\text { sation }\end{array}$ & disparition & $\begin{array}{l}\text { face à l'info, } \\
\text { seul }\end{array}$ & $\begin{array}{l}\text { brève, filet, } \\
\text { mouture }\end{array}$ & $\begin{array}{l}\text { fonctionnelle } \\
\text { ou d'utilité }\end{array}$ \\
\hline
\end{tabular}

\section{Proximité et identité éditoriale : le cas des magazines bretons}

Si notre démonstration, en deuxième partie, visait les propriétés intrinsèques des genres, nous proposons maintenant de voir comment les identités éditoriales et les conditions de production agissent sur la gestion des genres. Eu égard au corpus choisi, nous nous limiterons au croisement de la proximité géographique et de la proximité thématique, formule qui associe l'information magazine à un ciblage territorial. La presse quotidienne et hebdomadaire régionale a fait l'objet de plusieurs études menées par la composante lannionnaise du CRAPE ${ }^{21}$, mais nous avons choisi ici d'analyser la presse magazine régionale, soit Armor, Nouvel Ouest et ArMen.

Par rapport à la presse de proximité qui occupe le terrain de l'information locale, générale, quotidienne ou hebdomadaire ${ }^{22}$, comment se situent ces

21. Voir Y. Rochard, D. Ruellan, 1996, Un journalisme au coin du feu, document interne, IUT de Lannion; D. Ruellan, D. Thierry, 1998, Journal local et réseaux informatiques. Travail coopératif, décentralisation et identité des journalistes, Paris, L'Harmattan; B. Damian, R. Ringoot, D. Ruellan, D. Thierry, 2002, Information.loc@l, Paris, L'Harmattan.

22. La Bretagne a ceci de particulier qu'elle compte un nombre important de titres, dont deux quotidiens en concurrence : Ouest France et Le Télégramme. 
magazines régionaux qui n'ont au départ ni les moyens ni le professionnalisme de grands groupes de presse 23 ? La périodicité et la formule magazine les démarquent de la presse régionale quotidienne ou hebdomadaire et si l'ancrage territorial est aussi au cœur du projet éditorial, il est tout de même différent : le territoire n'est plus cette surface segmentée en zones d'information que sont les éditions locales, la proximité n'est plus forcément la mise en présence d'une actualité microlocale. Alors que la presse de proximité produit un journal géographiquement adapté au lecteur (sa ville, son canton, son département, sa région), la presse magazine de région postule un territoire compact et non morcelé. L'enjeu est en quelque sorte inversé. En presse régionale quotidienne, l'ampleur des zones de diffusion des quotidiens et le quadrillage en microsecteurs ont pour effet de produire un territoire hiérarchisé : la segmentation renvoie au découpage administratif (commune, canton, département...) et au découpage économico-médiatique (zones d'édition, zones de diffusion) ${ }^{24}$. Le territoire à géométrie variable pour nos trois magazines n'est pas un territoire organique, mais un territoire essentiel. Armor célèbre le génie breton à multiples facettes, Nouvel Ouest célèbre la puissance des «bâtisseurs » bretons, ArMen célèbre les savoirs et savoir-faire bretons. Ces traits identitaires se construisent en fonction d'un antisujet ou d'un antiprogramme (au sens greimassien) avec lequel ils entrent en conflit : Armor déplore le jacobinisme bridant l'épanouissement de tant de talents, Nouvel Ouest stigmatise les freins étouffant l'entreprise bretonne, ArMen lutte contre l'oubli organisé en appelant au travail de mémoire.

La proximité s'articule sur l'interprétation du territoire qui renvoie à de l'idéologie, mais aussi à du pragmatisme : pour Armor, la communauté est imaginée ${ }^{25}$ à partir de l'être et du ressenti bretons et l'ancrage régional est associé à un vision politique fédéraliste; pour Nouvel Ouest, la communauté est imaginée en fonction du faire et de la performance bretonne, et l'ancrage régional est associé à une vision économique ; pour ArMen, la communauté est imaginée en fonction du savoir et de la mémoire, et l'ancrage régional est associé à une vision encyclopédique de la Bretagne. Les lignes éditoriales ciblent le politique (Armor), l'économique (Nouvel Ouest) et l'historique (ArMen). Mais la grandeur des projets éditoriaux butte sur les moyens à mettre en œuvre, car les équipes

23. En personnel permanent : un directeur, un rédacteur en chef, un journaliste pour Armor; un rédacteur en chef, un secrétaire d'édition, un journaliste, un directeur pour Nouvel Ouest; un rédacteur en chef, 2,5 postes de rédacteur pour ArMen.

24. Voir R. Ringoot, J.-M. Utard, 2003, «Les élections européennes dans la presse régionale», dans É. Dacheux (dir.), L'Europe qui se construit, réflexions sur l'espace public européen, Publications universitaires de Saint-Étienne; R. Ringoot, J.-M. Utard, 2004, «L'Europe vue par la presse quotidienne régionale», dans D. Marchetti, É. Neveu (dir.), En quête d'Europe, médias européens et médiatisation de l'Europe, Presses universitaires de Rennes.

25. La communauté imaginée fait référence à B. Anderson. 
rédactionnelles sont très restreintes et/ou peu professionnalisées. Aussi faiton appel aux acteurs du champ prescrits par la ligne éditoriale : acteurs institutionnels, élus, administratifs, acteurs économiques (Armor, Nouvel Ouest), historiens ou experts (ArMen). Les genres dominants ou privilégiés sont respectivement l'éditorial, l'interview, l'enquête, mais les rédacteurs ne sont pas obligatoirement des journalistes professionnels. Conjointement à ces genres corporalisants et caractérisants dont l'usage n'est pas normatif, nous trouvons les genres dépersonnalisants, mais aussi et surtout des genres hybrides dans Armor et Nouvel Ouest, produits par les services de communication des organismes concernés : publi-rédactionnels, communiqués ou dossiers de presse, documents institutionnels reversés dans le journal sans traitement journalistique. Ce qui est remarquable, c'est que les trois magazines mettent leurs genres privilégiés au service de success-stories déclinées en fonction du style de chaque titre. À tel point que nous sommes tentés de voir là un facteur structurant le discours de la presse magazine régionale. Regardons plus en détail ce qu’il en est pour chaque magazine.

\section{Armor}

Le premier numéro du mensuel d'actualité Armor magazine, édité par la Sopel, a paru en mars 1969 à Paris. Son fondateur, Yann Poilvet, était le rédacteur en chef de La Vie bretonne, l'organe mensuel du Célib (Comité d'étude et de liaison des intérêts bretons), qui cessera de paraitre fin 1969. Très vite, il s'installe en Bretagne, à Saint-Brieuc. La revue, devenue simplement Armor («la mer» ou «le pays de la mer»), est aujourd'hui installée à Lamballe. Le directeur en est toujours Yann Poilvet. Sa fille Marie-Édith Poilvet en est la rédactrice en chef. Un journaliste salarié complète la rédaction. Depuis l'origine, les capitaux, modestes, sont familiaux. Le niveau des ventes réelles d'Armor est difficile à évaluer car sa diffusion n'est pas officiellement contrôlée (tirage annoncé : 40000 exemplaires). Plus de trente ans après sa création, la filiation de la revue avec l'organe d'information du Célib est toujours réelle et explique la collaboration régulière à la rédaction d'un certain nombre d'acteurs régionaux de la vie politique, sociale et économique. La ligne éditoriale est clairement régionaliste, vigoureusement antijacobine; la page consacrée à l'éditorial du directeur fondateur le rappelle chaque mois et prend une tonalité particulièrement offensive en périodes électorales.

L'éditorial est le genre privilégié en rubrique «politique» et «société». Partant presque systématiquement d'une information à caractère national, voire international, l'éditorial du directeur en vient inévitablement à la Bretagne pour s'en prendre aux méfaits du centralisme, réclamer une autonomie régionale 
accrue et en appeler à l'unité des Bretons au-delà des clivages politiques. Dans son sillage, plusieurs chroniques signées de collaborateurs réguliers, la plupart non journalistes, s'apparentent au genre éditorial : bâties sur l'argumentation, l'humeur et/ou la prise de position, elles ont pour objectif d'obtenir l'adhésion intellectuelle du lecteur, de conforter le positionnement politique du mensuel. En rubrique économique, on s'écarte de ce genre caractérisant pour faire place, très largement, à la citation qui révèle l'entretien avec une source extérieure et positionne l'auteur de l'article en médiateur. Il ne s'agit pourtant pas, à proprement parler, de reportage. Il n'y a ni mise en scène de la personne concernée, ni description, ni ambiance, ni ébauches de portrait; seulement le contenu d'un entretien restitué sous forme de citations ou d'appropriation par le journaliste, les premières représentant couramment les deux tiers ou les trois quarts du volume de l'article. S'agit-il de corporalisation ou de face à face entre lecteurs et informations ? Certes, le lecteur peut difficilement douter qu'il y ait eu rencontre, sur le terrain ou par téléphone, avec la source (la place accordée à cette dernière est comparable à celle que permet l'interview), mais l'énonciateur s'efface devant la source, la plaçant résolument au premier plan, réduisant la corporalisation du journaliste au profit de celle de la source, mettant en quelque sorte le lecteur face à face avec l'informateur. C'est bien la proximité entre sources et lecteur que le journal développe.

Ces articles essentiellement construits autour de citations d'une source - unique dans la plupart des cas - abondent également dans les dossiers consacrés aux villes et pays, dans les success-stories qui célèbrent le dynamisme de ces territoires, la pugnacité de leurs élus, le dynamisme de leurs entrepreneurs, le talent de leurs créateurs... Bâtis sous la forme d'un puzzle d'articles courts (en règle générale de 1200 à 2500 signes), ces success-stories abordent le territoire choisi de manière multithématique (économie, social, entreprise, formation, culture, art, initiatives, transports...) en évitant tout sujet non consensuel.

Seul genre journalistique présent (sauf à de rares exceptions) aux côtés de ces articles-citations, largement majoritaires dans les dossiers : l'interview, de préférence réservée aux maires et aux personnalités de premier plan. Intervenant en ouverture du dossier, cette incarnation forte du journaliste, représentant le titre, et du notable local ou régional vient créer d'emblée la proximité recherchée avec le lecteur. Success-story oblige, les questions posées sont ouvertes et offrent à l'interviewé un espace d'expression sans contrainte ni contradiction. 


\section{Nouvel Ouest}

Ce mensuel, à l'origine bimestriel, a été lancé en janvier 1998 par Hervé Louboutin, ancien rédacteur en chef de Presse-Océan, quotidien de Loire-Atlantique et de Vendée appartenant au groupe Hersant. Nouvel Ouest a choisi comme territoire d'information et de diffusion quatre "régions maritimes»: Bretagne, Pays de la Loire, Basse-Normandie, Poitou-Charente. En réalité, seules les deux premières sont couvertes en totalité, sans que l'on sache s'il s'agit d'un manque de moyens ou d'une difficulté de définition d'un territoire cohérent. Il s'agit, lit-on sous la plume du fondateur, de «faire travailler ensemble des régions qui veulent se donner la main pour résister au centralisme ambiant en se donnant les moyens de dominer elles-mêmes leur destin ". L'analogie avec le régionalisme d'Armor ou celui d'ArMen se limite à ce rejet du centralisme. La démarche des deux premiers est largement fondée sur la volonté d'affirmer et de développer une identité culturelle pour la Bretagne historique (génératrice pour Armor d'un projet politique fédéraliste), tandis que celle de Nouvel Ouest s'appuie sur un projet politique et économique néolibéral. Ses adversaires sont tous ceux qui lui apparaissent comme des obstacles à la liberté des entrepreneurs. La revue a plusieurs fois connu des difficultés financières. Le quotidien Le Télégramme fait partie des quelques partenaires d'Hervé Louboutin, principal actionnaire. Ses ventes, très modestes, ne sont pas contrôlées par l'Office de justification de la diffusion (tirage annoncé : 20000).

Bien que plus politique qu'Armor, au sens hexagonal du terme ${ }^{26}$, plus polémique aussi, Nouvel Ouest fait également la part belle aux success-stories. Une douzaine de pages, parfois plus, rassemblées dans une rubrique "Réussite», sont consacrées à l'histoire ou au portrait d'entreprises implantées sur sa zone de diffusion. Lorsque l'entreprise s'inscrit dans le patrimoine local ou régional, que deux générations au moins de dirigeants d'une même famille s'y sont succédé, le traitement se fait alors sous forme de dossiers intitulés «Saga».

Dans la rubrique «Réussite», le texte occupe généralement une double page. Selon des critères qui semblent relever plus d'éléments conjoncturels que de choix véritables, ils font appel à deux genres différents : l'interview et la mouture. Dans le premier cas, l'interviewé est le chef d'entreprise ou, à défaut,

26. Tandis qu'Armor Magazine néglige les divergences politiques internes à la région et se concentre sur les éléments de convergence, Nouvel Ouest affiche avec vigueur une vision politique et économique ultralibérale qui met en relief les lignes de fracture à l'intérieur même de la région. 
l'un de ses collaborateurs les plus proches. Les questions lui permettent de s'exprimer sans aucune contrainte ni contradiction. Comme le titre de la rubrique l'annonce, l'intéressé est invité à commenter sa réussite. Le résultat ne diffère guère du contenu habituel d'un document de communication externe d'entreprise. Dans le second cas (la mouture), selon un procédé presque systématique, l'article se compose d'informations puisées, d'une part, dans un dossier de presse ou dans des articles de presse, d'autre part, dans un entretien avec le responsable de l'entreprise ou l'un de ses adjoints. Si la personne interrogée par l'auteur de l'article est clairement identifiée, les autres sources utilisées ne sont quasiment jamais citées. Pour autant, il ne fait aucun doute pour le lecteur que les éléments d'information sont puisés dans des discours préexistants.

Dans le dossier "Saga», le journaliste puise sa matière dans la littérature produite autour de l'entreprise (articles historiques ou économiques, livres, dossiers de presse) et dans un ou plusieurs entretiens réalisés par téléphone, fax ou mail. En règle générale, les sources, à l'exception des personnes citées, sont passées sous silence, même lorsqu'il s'agit d'un travail d'investigation approfondi (fascicule, livre); elles peuvent donc être confondues avec l'auteur de l'article : l'effacement énonciatif des sources bénéficie alors à l'énonciateur, aussi discret soit-il.

\section{ArMen}

ArMen est une revue culturelle bimestrielle créée en février 1986, à Douarnenez (29), par la Scop Le Chasse-Marée, dans le sillage de la revue d'ethnologie maritime éponyme lancée en 1981. Dans sa première livraison, ArMen, soustitrée La Bretagne, un monde à découvrir, se présentait comme " une nouvelle revue, belle, moderne, documentée pour redécouvrir la Bretagne, du passé le plus lointain aux préoccupations d'aujourd'hui », qui entendait traiter d'histoire, d'économie, d'habitat, d'environnement, d'ethnologie, d'archéologie, d'art et de littérature. Ses fondateurs, qui avaient associé à leur réflexion plusieurs chercheurs de l'université de Bretagne Ouest, entendaient bâtir un «magazine territorial» grand public, scientifiquement rigoureux mais soucieux d'éviter le jargon des spécialistes. Le territoire concerné est celui de la Bretagne historique, associant donc la Loire-Atlantique aux quatre départements de la Bretagne administrative.

Lancée avec un minimum de moyens, ArMen a trouvé son lectorat dès les premiers numéros. Les ventes ont rapidement dépassé le cap des 10000 exemplaires, dont une large majorité d'abonnés d'une fidélité exceptionnelle (le taux de réabonnement a longtemps dépassé $90 \%$ ). Les ventes sont aujour- 
d'hui de l'ordre de 10500 exemplaires dont 7500 par abonnement. La rédaction se compose de deux journalistes assistés d'une secrétaire de rédaction et fait appel à deux catégories de collaborateurs occasionnels : des journalistes pigistes et des spécialistes intervenant dans leur domaine de compétence (historiens, économistes, géographes, naturalistes, etc.). Début 2003, à la suite de difficultés économiques de la Scop éditrice, ArMen a été reprise par les Éditions Fitament, un petit groupe de presse basé à Telgruc-sur-mer (29).

La success-story et la saga occupent une place de choix dans ArMen, mais sur un registre sensiblement différent. Elles relatent l'histoire d'une entreprise, d'un individu, d'un groupe humain présentés comme exemplaires et révélateurs du dynamisme politique, économique, social et culturel de la société bretonne d'hier et d'aujourd'hui.

Le genre utilisé est l'enquête. Le travail de recherche approfondi mené en amont apparait clairement dans les références aux documents d'archives, aux ouvrages divers, aux travaux universitaires. Il est complété par un réel travail de terrain auprès de multiples interlocuteurs, présents dans les nombreuses citations. Le journaliste se place dans une perspective historique. II se comporte en témoin d'une histoire en train de se faire. Il s'introduit dans les espaces laissés plus ou moins inexploités par les historiens, les ethnologues, les sociologues, et ce n'est pas un hasard si, fréquemment, ces derniers citent la revue en référence. La petite histoire, celle des entrepreneurs locaux, des savoir-faire séculaires, des traditions enracinées, vient nourrir la grande.

Dans ces articles longs de 15000 à 25000 signes, deux postures se mêlent étroitement et se complètent : la mise en scène du journaliste sur le terrain (portraits, citations, descriptions) et la référence aux discours préexistants (extraits d'études, de récits, d'analyses préexistantes). En fait, dans ArMen, le journaliste utilise les discours préexistants de la même manière qu'il met en scène son propre travail de terrain. Les sources - ouvrages d'histoire, d'ethnographie, récits de voyageurs... - sont scrupuleusement citées; leurs auteurs sont convoqués au même titre que les interlocuteurs rencontrés sur le terrain. Dans ArMen, qui se présente comme une "véritable encyclopédie vivante de la Bretagne et des pays celtiques», le journaliste s'efface derrière le spécialiste en usant largement de la citation-référence. Il faut aussi préciser que, pendant longtemps, les journalistes de la rédaction n'ont pas signé leurs textes. La majorité des articles publiés est d'ailleurs l'œuvre d'experts, et les journalistes qui interviennent sont souvent eux-mêmes spécialisés. 


\section{Success-story et gestion des genres journalistiques}

Malgré leurs différences évidentes (esthétique éditoriale, contenus, traitements), ces magazines présentent aussi des convergences. Pour Armor et Nouvel Ouest, le vecteur d'identité territoriale semble neutraliser la tension entre communication et information, comme l'indique l'utilisation des sources communicationnelles et institutionnelles et le positionnement journalistique effacé quand il y a intervention du journaliste. Ce brouillage entre information et communication est peut-être le fait du manque de moyens, notamment en personnel, mais nous trouvons aussi ce phénomène en structures très professionnalisées : les évènements culturels sont souvent traités en presse régionale quotidienne sous forme de promotion du territoire; nous pensons particulièrement aux suppléments consacrés aux manifestations sportives et artistiques. Il faut donc s'interroger sur les dérives potentielles ou avérées d'un «patriotisme local $»^{27}$ touchant les titres régionaux en général. Si l'on peut imputer cette posture à des contraintes commerciales ou politiques, il n'est pas dit que les pressions économiques ou institutionnelles soient les seuls facteurs déterminants. Une forme de militantisme de terroir est systématiquement développée dans la presse territoriale. Cette solidarité communautaire est tellement intériorisée par les journalistes (et qui plus est par des rédacteurs non professionnels) que la tendance à valoriser le territoire est soit inconsciente, soit ramenée à «la bonne cause». De la proximité à l'empathie, il n'y a qu'un pas.

Le point de convergence concernant les trois magazines relève du même phénomène : la success-story comme orientation éditoriale dénote l'incontournable éloge du territoire à travers ses "héros». Ces derniers, convoqués en tant que sources dans Armor et Nouvel Ouest, sont «corporalisés» et/ou «caractérisés» au détriment du journaliste réduit à la portion énonciative congrue. Dans les deux titres, on use du discours rapporté de personnes sources en l'autonomisant de manière à évacuer tout marqueur circonstanciel, et du discours rapporté de documents sources en évitant de les référencer. Cette prédominance de la proximité entre lecteur et sources est corrélée à un usage des genres peu normatif : les écrits communicationnels (transmis par les relations presse) sont dilués dans le journal et les genres journalistiques sont mixtes : interview-commentaire ou mouture de Nouvel Ouest, reportage-entretien et article-citation d'Armor.

27. Cette expression empruntée à D. Ruellan a été formulée lors d'une conférence-débat intitulée "La presse quotidienne en Bretagne», organisée par Y. Rochard pour le Groupement d'action culturelle de Landernau, le 30 octobre 2003 à Landernau, Finistère. 
La success-story d'ArMen fonctionne très différemment... Si l'éloge de la région par ses confrères est basé sur la monstration de personnages «ostentatoires ", l'éloge par ArMen est basé sur la démonstration. On remarque ici aussi une hybridation de genre entre enquête journalistique et enquête historique qui dénote bien le projet. Ici on milite pour le savoir, rédacteurs, sources et lecteurs, comme le confirme le taux d'abonnement. Et si la valorisation des sources humaines est équivalente à la valorisation des sources documentaires, c'est bien parce que la mise en scène du savoir en train de se construire est tout aussi importante que le contenu du savoir diffusé.

La success-story comme emblème de la communauté régionale imaginée par les magazines bretons s'articule sur des genres corporalisants distribués soit en rubrique économique (Nouvel Ouest), soit dans des «dossiers» (Armor, ArMen, Nouvel Ouest), en s'accordant sur l'idée que le dossier traite un seul sujet d'information mais sous plusieurs angles, avec un volume et une iconographie plus importants qu'une simple rubrique. La success-story est donc associée à une valorisation particulière de l'information. Elle est traitée soit par l'interview sous sa forme classique, soit par des articles privilégiant le discours rapporté rappelant les entretiens préalables, soit par l'enquête (ArMen). Mais globalement, ce processus de corporalisation concerne moins le journaliste que les sources : effacement énonciatif du rédacteur dans la restitution d'entretiens, positionnement minimal du journaliste dans les questions d'interviews. Seule l'enquête d'ArMen corporalise tout autant le rédacteur que ses sources.

Par ailleurs, quand il y a recours au genre dépersonnalisant (la mouture de Nouvel Ouest), la source est tout de même mise en relief par le biais de citations.

Par rapport à notre proposition normative préalable, nous pouvons statuer que les magazines bretons développent un genre de proximité physique et émotionnelle, mais que celui-ci s'articule sur la corporalisation des sources. La mise en scène de la réussite bretonne se construit sur un ethos corporalisé de ses acteurs, ce qui revient à construire un ethos breton (compétent, gagnant, créateur...). Même ArMen, qui met en évidence le travail du journaliste (voire de l'historien : investigation, analyse, entretiens, documents) travaille à la gloire de la culture bretonne par son contenu mais aussi par sa propre action.

Si la proximité éditoriale en journalisme est issue de l'interaction entre journaliste / source / public, elle est aussi inféodée aux types d'appartenance définis par les journaux. Aussi, la gestion de la distance représente un enjeu majeur dans l'équilibre à trouver entre professionnalisme journalistique et communauté imaginée. Partage, identification, complicité, adhésion, communion, 
circularité, séduction, communautarisation ${ }^{28}$, socialisation, intériorisation, sont autant d'expressions traduisant des manières d'être proches issues de l'interaction entre la proximité induite par les genres (émotionnelle, physique, intellectuelle, fonctionnelle) et la proximité travaillée par la ligne éditoriale, quelle que soit l'appartenance revendiquée.

28. F. Le Cam, doctorante CRAPE, analyste des weblogs d'information éthique, pose l'éventualité de l'émergence d'une pratique journalistique communautaire caractérisant ces sites d'autopublication. "Nouvelles pratiques d'information : extension ou dérive du journalisme ? », Journées d'étude, Strasbourg, 24-25 octobre 2003. 